\title{
Release of ATP in the Ventral Medulla during Hypoxia in Rats: Role in Hypoxic Ventilatory Response
}

\author{
Alexander V. Gourine, ${ }^{1}$ Enrique Llaudet, ${ }^{2}$ Nicholas Dale, ${ }^{2}$ and K. Michael Spyer ${ }^{1}$ \\ ${ }^{1}$ Department of Physiology, Royal Free and University College Medical School, London NW3 2PF, United Kingdom, and ${ }^{2}$ Warwick Biosensor Group, \\ Department of Biological Sciences, University of Warwick, Coventry CV4 7AL, United Kingdom
}

$\mathrm{P}_{2} \mathrm{X}_{2}$ receptor subunits of the ATP-gated ion channels are expressed by physiologically identified respiratory neurons in the ventral respiratory column, implicating ATP in the control of respiratory activity. We now show that, during hypoxia, release of ATP in the ventrolateral medulla (VLM) plays an important role in the hypoxic ventilatory response in rats. By measuring ATP release in real time at the ventral surface of the medulla with novel amperometric biosensors, we found that hypoxia $\left(10 \% \mathrm{O}_{2} ; 5\right.$ min $)$ induced a marked increase in the concentration of ATP $(\sim 3 \mu \mathrm{M})$. This ATP release occurred after the initiation of enhanced respiratory activity but coincided with the later hypoxia-induced slowing of the respiratory rhythm. ATP was also released at the ventral surface of the medulla during hypoxia in peripherally chemodenervated animals (vagi, aortic, and carotid sinus nerve sectioned). By using horizontal slices of the rat medulla, we found that, during hypoxia, ATP is produced throughout the VLM in the locations corresponding to the ventral respiratory column. Blockade of ATP receptors in the VLM (microinjection of P2 receptor antagonist pyridoxal-5' -phosphate-6-azophenyl-2' $4^{\prime}$-disulphonic acid; $100 \mu \mathrm{M}$ ) augmented the hypoxia-induced secondary slowing of the respiratory rhythm. Our findings suggest that ATP released within the ventral respiratory column is involved in maintenance of the respiratory activity in conditions when hypoxia-induced slowing of respiration occurs. These data illustrate a new functional role for ATP-mediated purinergic signaling in the medullary mechanisms controlling respiratory activity.

Key words: ATP; biosensor; hypoxia; P2X; respiration; ventrolateral medulla

\section{Introduction}

The CNS of animals with high metabolic rates (mammals and birds) is extremely sensitive to a decrease in $\mathrm{P}_{\mathrm{O}_{2}}$ in the arterial blood (i.e., hypoxia). Oxygen sensors of the carotid bodies, located strategically at the bifurcation of the common carotid artery, have evolved to monitor $\mathrm{P}_{\mathrm{O}_{2}}$ in the blood entering the brain and to initiate adaptive changes in respiratory activity when hypoxia occurs (Gonzalez et al., 1994; Daly, 1997; Prabhakar, 2000). The hypoxic ventilatory response in adults consists of an initial increase in respiration, followed by a secondary slowing of the respiratory rhythm, known as the hypoxic ventilatory depression (Richter et al., 1991; Bissonnette, 2000). We showed recently that, within the carotid body, ATP released by the $\mathrm{O}_{2}$-sensitive glomus cells and acting at $\mathrm{P} 2 \mathrm{X}_{2}$ receptors located on the afferent terminals of the carotid sinus nerve forms an essential link in peripheral chemosensory transduction, leading to the enhancement of ventilation during hypoxia (Rong et al., 2003).

The mechanisms responsible for the hypoxia-induced secondary slowing of the respiratory rhythm are less well understood (for review, see Bissonnette, 2000). Adenosine has been impli-

Received Sept. 10, 2004; revised Dec. 3, 2004; accepted Dec. 4, 2004

This work was supported by Biotechnology and Biological Sciences Research Council (K.M.S.) and The Wellcome Trust (N.D.).

Correspondence should be addressed to Dr. Alexander V. Gourine, Department of Physiology, Royal Free and University College Medical School, Rowland Hill Street, London NW3 2PF, UK. E-mail: a.gourine@rrc.ucl.ac.uk. D0I:10.1523/JNEUROSCI.3763-04.2005

Copyright $\odot 2005$ Society for Neuroscience $\quad 0270-6474 / 05 / 251211-08 \$ 15.00 / 0$ cated as a potential mediator (Runold et al., 1989; Thomas and Marshall, 1994; Schmidt et al., 1995; Chau and Koos, 1999; Johansson et al., 2001). However, we recently demonstrated that adenosine release in the medullary regions involved in respiratory control, nucleus tractus solitarii and rostral ventrolateral medulla (VLM), occurs too late to be responsible for the initiation of hypoxia-induced depression of the respiratory activity (Gourine et al., 2002). Interestingly, the ecto-5' -nucleotidase inhibitor $\alpha, \beta$-methylene ADP greatly reduced the release of adenosine during hypoxia at the dorsal surface of the medulla, suggesting that it is produced in the extracellular space by the previous release and breakdown of ATP (Gourine et al., 2002). Furthermore, we recently found that $\mathrm{P} 2 \mathrm{X}_{2}$-deficient mice exhibited a much more profound hypoxia-induced ventilatory depression compared with their wild-type counterparts (Rong et al., 2003), suggesting that the action of ATP on $\mathrm{P} 2 \mathrm{X}_{2}$ receptors in either the brain or the periphery may oppose ventilatory depression.

Ionotropic P2X receptors of the ATP-gated ion channel (North, 2002) are present in areas of the VLM corresponding to the ventral respiratory column (Kanjhan et al., 1999; Yao et al., 2000, 2003; Thomas et al., 2001), which is responsible for generation and patterning of the respiratory rhythm. More specifically, we demonstrated that a significant proportion of the VLM respiratory neurons express the $\mathrm{P}_{2} \mathrm{X}_{2}$ receptor subunit and increase their discharge in response to ATP (Gourine et al., 2003). Our previous studies also demonstrated that ATP contributes to the 
excitation of VLM inspiratory neurons during hypercapnia (for review, see Spyer and Thomas, 2000). We proposed that ATPmediated purinergic signaling at the level of the ventral respiratory column may also play an important role in the respiratory response to hypoxia and have therefore investigated this possible role. Here, we report that ATP is released in the VLM in response to hypoxia. This release of ATP at locations corresponding to the ventral respiratory column helps to maintain respiratory activity at the times when hypoxia-induced slowing of the respiratory rhythm occurs.

\section{Materials and Methods}

\section{Surgical procedures}

Experiments were performed on 31 male Sprague Dawley rats (300-340 g) and were performed in accordance with the United Kingdom Animals (Scientific Procedures) Act of 1986. The rats were anesthetized with pentobarbitone sodium (Sagatal; 60 mg/kg, i.p.; Rhône Mérieux, Harlow, UK). Anesthesia was maintained with supplemental doses of pentobarbitone sodium injected intravenously as required $\left(10 \mathrm{mg} \cdot \mathrm{kg}^{-1} \cdot \mathrm{h}^{-1}\right)$. Adequate anesthesia was ensured by maintaining stable levels of arterial blood pressure $(\mathrm{ABP})$, heart, and central respiratory rates and monitored by the absence of a withdrawal response to a paw pinch. The femoral artery and vein were cannulated for measurement of ABP and administration of anesthetic, respectively. The trachea was cannulated, and the animal was ventilated with a mixture of $50 \% \mathrm{O}_{2}$ and $50 \% \mathrm{~N}_{2}$ (unless otherwise required by the protocol) using a positive pressure ventilator (Harvard rodent ventilator; model 683; Harvard Apparatus, Holliston, MA) with a tidal volume of $\sim 2 \mathrm{ml}$ and a ventilator frequency similar to the normal respiratory frequency ( $\sim 60$ strokes $/ \mathrm{min})$. The animal was then paralyzed with a neuromuscular blocker (gallamine triethiodide; Flaxedil; $10 \mathrm{mg} / \mathrm{kg}$, i.v.; maintained with supplemental doses of 1-2 $\mathrm{mg} \cdot \mathrm{kg}^{-1} \cdot \mathrm{h}^{-1}$, i.v.) and was placed in a stereotaxic frame. Either the ventral or dorsal surface of the brainstem was exposed. To expose the dorsal medullary surface, an occipital craniotomy was performed, and the cerebellum was partially removed. The exposed area of the brain was protected by covering with modified Bulmer's buffer, which consisted of the following (in mM): $100 \mathrm{NaCl}, 1 \mathrm{MgSO}_{4}, 2 \mathrm{KP}_{\mathrm{i}}$ buffer, and 5 glycerol, $\mathrm{pH} 7.4$.

In five rats, peripheral chemodenervation was performed; the vagi and the aortic nerves were sectioned bilaterally, and the carotid sinus nerves were transected bilaterally at the junction with the glossopharyngeal trunk.

Activity of the phrenic nerve was recorded as an indicator of central respiratory drive. The signal was amplified $(20,000 \times)$, filtered (500$1500 \mathrm{~Hz})$, and rectified and smoothed $(\tau=50 \mathrm{~ms})$. Partial pressures of $\mathrm{O}_{2}$ and $\mathrm{CO}_{2}$, as well as $\mathrm{pH}$ of the arterial blood, were measured every 1-2 h. End-tidal levels of $\mathrm{CO}_{2}$ were monitored on-line using a fast-response $\mathrm{CO}_{2}$ analyzer (CapStar-100; CWE, Ardmore, $\mathrm{PA}$ ) and kept at designated levels (either above or below the apneic threshold) by altering tidal volume and respiratory frequency. The body temperature was maintained with a servo-controlled heating pad at $37.0 \pm 0.2^{\circ} \mathrm{C}$.

\section{Slice preparation for in vitro experiments}

Young adult rats ( $\sim 5$ weeks of age; $100-120 \mathrm{~g} ; n=45)$ were killed by cervical dislocation, and the brainstem was quickly removed from the skull and placed in artificial CSF (aCSF) chilled to $\sim 5^{\circ} \mathrm{C}: 124 \mathrm{~mm} \mathrm{NaCl}$, $3 \mathrm{~mm} \mathrm{KCl}, 2 \mathrm{~mm} \mathrm{CaCl}, 26 \mathrm{~mm} \mathrm{NaHCO}_{3}, 1.25 \mathrm{~mm} \mathrm{NaH}_{2} \mathrm{PO}_{4}, 1 \mathrm{~mm}$ $\mathrm{MgSO}_{4}$, and $10 \mathrm{~mm}$ D-glucose saturated with $95 \% \mathrm{O}_{2}$ and $5 \% \mathrm{CO}_{2}, \mathrm{pH}$ 7.4 , with an additional $11 \mathrm{~mm} \mathrm{Mg}^{2+}$. The medulla oblongata was isolated, and the arachnoid matter was removed, but the pia was left in place. The isolated medulla was placed on a block, ventral side up, and horizontal slices of $400 \mu \mathrm{m}$ thickness were cut using a vibratome (see Fig. $4 a$ ). Once cut, the slices were stored in a chamber containing normal aCSF saturated with $95 \% \mathrm{O}_{2}$ and $5 \% \mathrm{CO}_{2}, \mathrm{pH} 7.4$, at room temperature. Recordings were made from the slices placed on an elevated grid in a flow chamber at $35^{\circ} \mathrm{C}$ to permit access of $\mathrm{O}_{2} / \mathrm{CO}_{2}$-saturated aCSF from both sides of the slice. Glycerol ( $2 \mathrm{~mm}$ ) essential for ATP biosensor operation (see below) was added to the aCSF. The rate of perfusion was $6 \mathrm{ml} / \mathrm{min}$. a

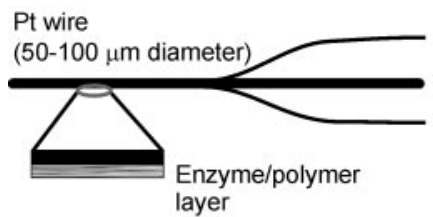

C

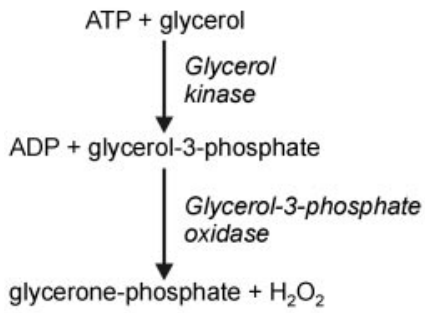

e

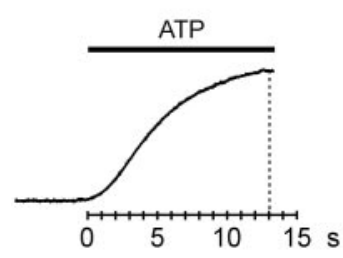

b

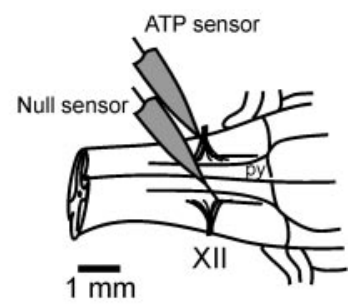

d

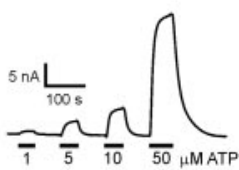

Figure 1. Principle and performance of ATP biosensors and their placement on the ventral surface of the medulla oblongata. $\boldsymbol{a}$, Scheme of the sensor assembly. An expanded portion of one side of the sensor is shown to indicate the enzymatic biolayer. Note that this biolayer completely surrounds the Pt wire. $\boldsymbol{b}$, Schematic showing of the sensor placement on the ventral surface of the medulla oblongata. The sensors were bent so that they could be placed parallel to and in contact with the exposed surface of the medulla. A dual recording configuration of the ATP sensor placed on one side of the medulla along with a null sensor that was placed in an equivalent position on the other side of the medulla was used. $c$, The enzymatic cascade used by the ATP biosensors. The presence of the target analyte ATP results in the production of $\mathrm{H}_{2} \mathrm{O}_{2}$, which is detected electrochemically. $\boldsymbol{d}$, Calibration curve of the ATP biosensor, demonstrating linearity of ATP detection in concentrations from 1 to $50 \mu \mathrm{m}$. Inset, Sample responses for the ATP biosensor. $\boldsymbol{e}$, An expanded portion of the calibration trace demonstrating the fast response characteristics of the ATP biosensor, which reaches full response within $10-13 \mathrm{~s}$. Changeover of the solution in the calibration chamber was not a step change. It would take a few seconds for ATP to reach the desired concentration. Therefore, the trace shows a speed of response that is a combination of the time taken for the change in ATP concentration in the chamber to occur and the response of the sensor. Note that the sensor responds immediately when ATP solution is starting to enter the chamber. $f$, Calibration trace showing that the ATP biosensors are insensitive to adenosine, UTP, or ADP. py, Pyramidal tract; XII, hypoglossal nerve roots.

\section{ATP biosensors}

The principal design and operation of the enzyme-based biosensors used in this study have been described in detail previously (Llaudet et al., 2003, 2004). The ATP sensors (Sarissa Biomedical, Coventry, UK) comprised two enzymes, glycerol kinase and glycerol-3-phosphate oxidase entrapped within a matrix around a fine platinum (Pt) wire of $100 \mu \mathrm{m}$ in diameter and $1 \mathrm{~mm}$ in length (Fig. 1). After fabrication, the sensors were bent so that they could be placed parallel to and in contact with the exposed surface of the medulla (Fig. 1b).

Electrochemical sensors can respond not only to the analyte of interest but also to any electroactive species in the millieu. To ensure that the sensors were responding specifically to ATP, we used a dual recording configuration of an ATP sensor placed on one side of the medulla, along with a null sensor lacking enzymes, which was placed in an equivalent position on the other side of the medulla (Fig. 1b). The null sensors thus served as a control to determine whether any "nonspecific" electroactive 
interferents were released and could confound the measurements. This dual recording configuration was used for all of the experiments.

The ATP sensors are virtually insensitive to ADP, UTP, or adenosine (Fig. $1 f$ ). They require the presence of glycerol to be active; however, their response is insensitive to variations in glycerol concentration over the range of $0.5-5 \mathrm{~mm}$. The magnitude of the response to ATP is also insensitive to changes in $\mathrm{pH}$.

Sensors were calibrated in vitro immediately before and after the recordings (some 3-4 h later) to test whether they retained sensitivity. For calibration, sensors were placed in a simple flow chamber that was continually perfused with control saline. The flow was switched to a saline containing $10 \mu \mathrm{M}$ ATP to determine the sensitivity of the sensor. During recordings in vivo, the sensors lost, on average, $\sim 70 \%$ of their initial sensitivity. Virtually no decrease in the responsiveness of the sensors to ATP occurred after their use in in vitro experiments. To convert changes in sensor current to changes in ATP concentrations, the mean of the initial and final calibrations was used.

\section{Experimental protocols}

Measurements of the hypoxia-induced changes in ATP concentration on the ventral surface of the medulla. The sensors were connected to a MicroC potentiostat (World Precision Instruments, Sarasota, FL) and held on a stereotaxic micromanipulator. The sensors were aligned with the pyramidal tracts and major blood vessels running on the ventral surface of the medulla and then were placed on the ventral surface $1.5-2.0 \mathrm{~mm}$ lateral from the midline (Fig. 1b). The sensitive part of the sensor was $\sim 1 \mathrm{~mm}$ in length and was placed in direct contact with a significant portion of the chemosensitive area on the ventrolateral medullary surface. The recording sites were located $\sim 400-700 \mu \mathrm{m}$ ventrally from the rostral divisions of the medullary ventral respiratory column (Bötzinger and preBötzinger complexes and the retrotrapezoid nucleus). For recordings from the dorsal surface of the medulla, sensors were aligned with the obex and placed $1.5 \mathrm{~mm}$ lateral to the midline so that its sensitive part spanned from the level of obex to $1 \mathrm{~mm}$ rostral of obex. The identical placement of the ATP sensors and null sensors was achieved by aligning them to the midline, pyramidal tracts (for recordings from the ventral surface) or obex (for recordings from the dorsal surface), landmark blood vessels, nerve roots, and by means of the vernier scale of the manipulator. Once the sensors were placed, the exposed area of the brainstem and both sensors were covered with an excessive volume of modified Bulmer's buffer (to provide glycerol for the operation of the sensor), and a period of 20-30 min was allowed, until a steady baseline was obtained. Note that the buffer would have been diluted by the CSF present on the ventral surface and by the constant outflow of the CSF from the exposed brainstem surface. However, ATP biosensor response is insensitive to variations in glycerol concentration over the range of $0.5-5 \mathrm{~mm}$.

Hypoxia was induced by ventilating the animals with a gas mixture containing $10 \% \mathrm{O}_{2}$ and $90 \% \mathrm{~N}_{2}$ for $5 \mathrm{~min}$. To determine the precise temporal relationship between changes in ATP concentration on the ventral surface of the medulla and hypoxia-evoked changes in the respiratory activity, in seven animals, apnea was induced by mechanically hyperventilating the animal so that $\mathrm{P}_{\mathrm{CO}_{2}}$ in the arterial blood and endtidal level of $\mathrm{CO}_{2}$ were under the apneic threshold. In these experiments, the hypoxic stimulus was the same $\left(10 \% \mathrm{O}_{2}\right.$ for $\left.5 \mathrm{~min}\right)$.

At the end of the experiment, five animals received pressure microinjections of pontamine sky blue dye ( $2 \%$ in $50 \mathrm{nl}$ of $0.2 \mathrm{M}$ sodium acetate) at two locations along the rostrocaudal extent of the ventrolateral medulla directly underneath the recording sites. These marked sites were identified histologically and mapped using a stereotaxic atlas (Paxinos and Watson, 1998). Histological analysis of the representative sensor placements confirmed that recording sites were within the targeted regions of the ventrolateral medullary surface.

In in vitro experiments, an analog of hypoxia was induced by perfusing the chamber with aCSF in which $\mathrm{O}_{2}$ had been replaced by bubbling the solution with $95 \% \mathrm{~N}_{2}$ and $5 \% \mathrm{CO}_{2}$. In the majority of experiments, changes in the $\mathrm{P}_{\mathrm{O}_{2}}$ of the perfusate were monitored on-line using a dissolved oxygen meter (model $\mathrm{ISO}_{2}$; World Precision Instruments). ATP and null sensors were placed in equivalent positions on the slice, as judged by the distance from the pyramidal tracts, the remaining nerve roots, and the edge of the slice.

Effect of P2 receptor blockade in the VLM on hypoxia-induced changes in respiratory activity. The rats $(n=7)$ were prepared as described above (see above, Surgical procedures). In addition, bilateral cervical vagotomy was performed, and the animal was placed in the stereotaxic frame dorsal side up. An occipital craniotomy was performed, and the cerebellum was partially removed to expose the dorsal surface of the brainstem. A threebarrelled glass micropipette (tip size, $20-25 \mu \mathrm{m}$ ) was placed in the area of rostral VLM [stereotaxic coordinates, $2.3 \mathrm{~mm}$ rostral to the calamus scriptorius, $2.0 \mathrm{~mm}$ lateral to midline, and $2.6-2.8 \mathrm{~mm}$ ventral from the dorsal surface of the medulla oblongata (Paxinos and Watson, 1998)]. The barrels of the micropipette contained P2 receptor antagonist pyridoxal-5' -phosphate-6-azophenyl-2', $4^{\prime}$-disulphonic acid (PPADS) (100 $\mu \mathrm{M}, \mathrm{pH}$ 7.4; Sigma, Poole, UK), vehicle [aCSF (in mM): $124 \mathrm{NaCl}$, $3.3 \mathrm{KCl}, 2.4 \mathrm{CaCl}_{2}, 1.3 \mathrm{MgSO}_{4}, 26 \mathrm{NaHCO}_{3}, 1.2 \mathrm{KH}_{2} \mathrm{PO}_{4}$, and 10 D-glucose], and pontamine sky blue dye ( $2 \%$ in $0.2 \mathrm{M}$ sodium acetate). The injections were made using pressure over $5-10 \mathrm{~s}$ and were monitored using a dissecting microscope with a calibrated micrometer disk.

In the preliminary studies, we determined that the second episode of hypoxia applied $>1 \mathrm{~h}$ after the first episode induces an equivalent hypoxic ventilatory response. Therefore, we used the same experimental animal to determine control hypoxic respiratory response after microinjection of aCSF into the VLM and to compare this response with that evoked by hypoxia after administration of PPADS at the same medullary site. First, unilateral microinjection of aCSF $(50 \mathrm{nl})$ was made into the left VLM. Five minutes later, hypoxia was induced by ventilating the animals with a gas mixture containing $10 \% \mathrm{O}_{2}$ and $90 \% \mathrm{~N}_{2}$ for $5 \mathrm{~min}$; thus, control hypoxic ventilatory response was obtained. Subsequently, the animal was ventilated normally for at least $1 \mathrm{~h}$ to reestablish physiological levels of $\mathrm{P}_{\mathrm{O}_{2}}, \mathrm{P}_{\mathrm{CO}_{2}}$, and $\mathrm{pH}$ in the arterial blood and to resume normal phrenic nerve activity. After this recovery period, PPADS (100 $\mu \mathrm{M} ; 50 \mathrm{nl})$ was microinjected into the same VLM site, and, after $5 \mathrm{~min}$, the respiratory response to hypoxia was tested again. Blood gases were measured during hypoxia episodes to ensure that arterial $\mathrm{P}_{\mathrm{O}_{2}}$ and $\mathrm{P}_{\mathrm{CO}_{2}}$ levels were similar in both cases. The sites of microinjections were marked by pressure injection of pontamine sky blue dye, identified histologically, and mapped using a stereotaxic atlas (Paxinos and Watson, 1998).

\section{Data analysis}

Records were processed and analyzed using Spike 2 software (Cambridge Electronic Design, Cambridge, UK). Changes in ATP levels are presented as means \pm SEM of peak (as a micromolar concentration) and integral (in micromolar concentration times seconds) increases in concentrations. Integral increases in ATP concentrations were determined by measuring area under the curve relative to a straight line joining the sensor current level before and after the response. The integral gives a better measure of the total production of analyte than the peak concentration.

Hypoxia-induced changes in the respiratory rate, amplitude of the phrenic nerve bursts, and minute ventilation (respiratory rate $\times$ amplitude of the phrenic nerve bursts) after injection of aCSF or PPADS into the VLM were compared using Student's paired $t$ test, and then the data were normalized for graphical purposes. A value of $p<0.05$ was considered significant.

\section{Results}

Release of ATP at the ventral surface of the medulla oblongata during hypoxia

During systemic hypoxia, the ATP microelectrode biosensors responded with a large increase in current, which occurred $25.0 \pm$ $4.5 \mathrm{~s}$ after hypoxia-induced activation of the respiratory activity (mean signal, $970 \pm 280 \mathrm{pA}$ and $293 \pm 87 \mathrm{nA} \cdot \mathrm{s} ; n=19$ ) (Figs. 2, $3)$. This large signal contrasted with that recorded by the "null" sensors that lacked enzymes in the polymer coating (mean signal, $380 \pm 120 \mathrm{pA}$ and $98 \pm 33 \mathrm{nA} \cdot \mathrm{s} ; n=18 ; p=0.021$ and $p=0.015$, respectively, vs ATP sensor) (Figs. 2, 3). We therefore concluded that the signal recorded during hypoxia by the ATP biosensors was attributable to the increase in ATP concentration in the area 
a

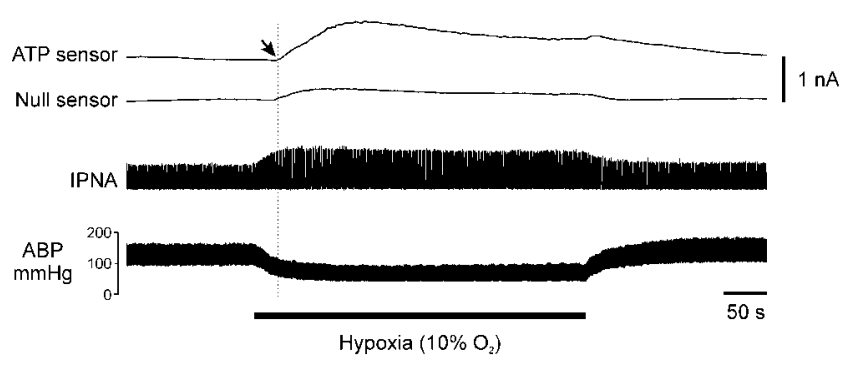

b
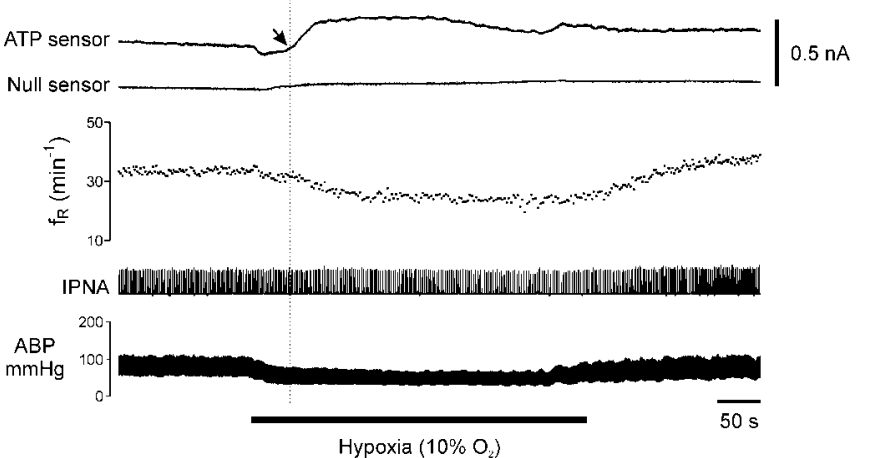

C

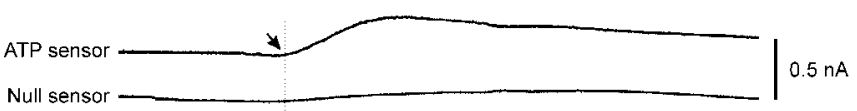

IPNA

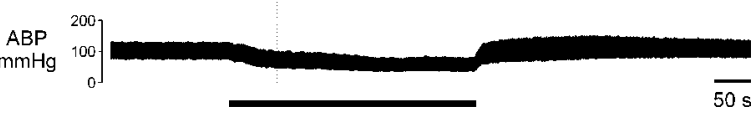

Hypoxia $\left(10 \% \mathrm{O}_{2}\right)$

Figure 2. Release of ATP at the ventral surface of the medulla oblongata during hypoxia in rats. $\boldsymbol{a}$, Representative raw data illustrating changes in the arterial blood pressure, phrenic nerve discharge, and null and ATP sensor currents during systemic hypoxia $\left(10 \% \mathrm{O}_{2}\right.$ in the inspired air). $\boldsymbol{b}$, Representative raw data illustrating changes in the arterial blood pressure, phrenic nerve discharge, and release of ATP at the ventral surface of the medulla during hypoxia in sinoaortically denervated and vagotomized animals. $c$, Release of ATP at the dorsal medullary surface: raw data illustrating changes in the arterial blood pressure, phrenic nerve discharge, and release of ATP at the dorsal surface of the medulla oblongata during hypoxia in rats. IPNA, Integrated phrenic nerve activity (arbitrary units); $f_{R}$, frequency of the phrenic nerve bursts. Arrows indicate the moment at which the concentration of ATP starts to increase above the baseline.

surrounding the sensor rather than to the generation of nonspecific electroactive interferents. Sometimes, ATP and/or null sensors exhibited a small negative shift around the onset of hypoxia (Figs. 2b, 3b). This is likely to be attributable to changes in $\mathrm{P}_{\mathrm{O}_{2}}$ in the neighborhood of the sensor, which consists of a Pt wire polarized to $500 \mathrm{mV}$ and therefore exhibits some intrinsic $\mathrm{O}_{2}$ sensitivity. Such shifts in sensor signal during hypoxia have been reported previously in experiments in vitro and in vivo (Dale et al., 2000; Gourine et al., 2002). We therefore do not consider this negative shift a fall in ATP concentration

We recorded a marked increase in ATP concentration on the ventrolateral surface of the medulla during systemic hypoxia (Fig. $2 a)$ that had a mean amplitude of $3.0 \pm 0.7 \mu \mathrm{M}(n=16)$. The total amount of ATP released during hypoxia was estimated to be $817 \pm 214 \mu \mathrm{M} \cdot \mathrm{s}(n=16)$. The increase in ATP concentration on

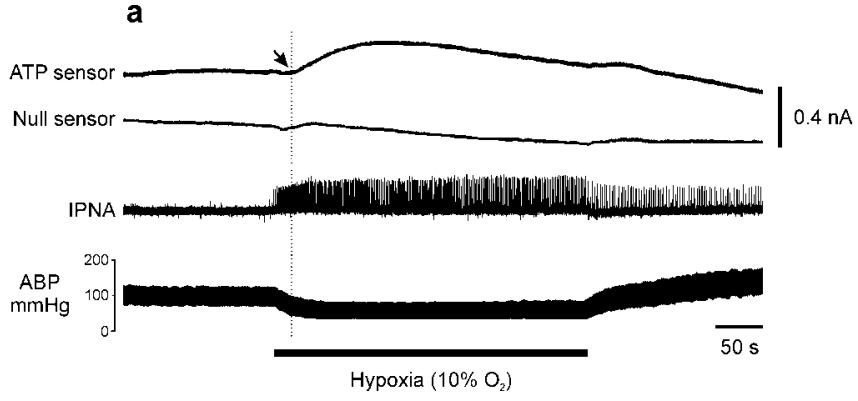

b

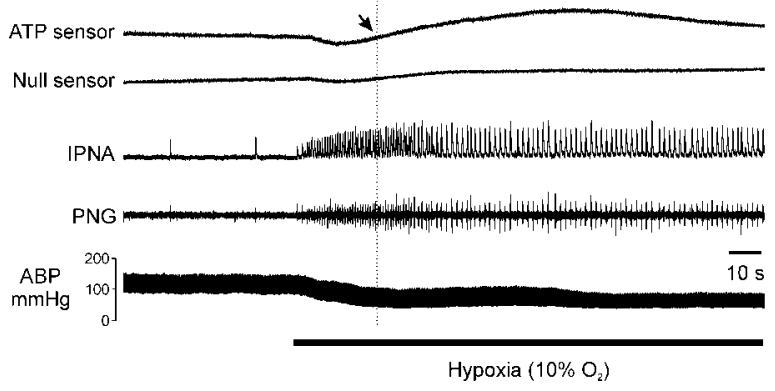

Figure 3. Release of ATP at the ventral surface of the medulla oblongata during hypoxia in rats that were mechanically hyperventilated to induce central apnea (arterial $\mathrm{P}_{\mathrm{CO}_{2}}$ and end-tidal $\mathrm{CO}_{2}$ below apneic threshold). $\boldsymbol{a}$, Representative raw data illustrating changes in the arterial blood pressure, phrenic nerve discharge, and null and ATP sensor currents during systemic hypoxia ( $10 \% \mathrm{O}_{2}$ in the inspired air). $\boldsymbol{b}$, An expanded portion of the raw data obtained in a different experiment, illustrating that, during hypoxia, an increase in ATP concentration on the ventral surface of the medulla occurs after the activation of the respiratory network and peaks during the secondary hypoxia-induced slowing of the respiratory rhythm. PNG, Phrenic neurogram; IPNA, integrated phrenic nerve activity (arbitrary units). Arrows indicate the moment at which the concentration of ATP starts to increase above the baseline.

the ventral surface of the medulla was delayed and occurred $25.0 \pm 4.5 \mathrm{~s}$ after hypoxia-induced augmentation of the respiratory activity. Experiments in animals in which apnea was induced by mechanical hyperventilation confirmed that, during hypoxia, release of ATP on the ventral surface of the medulla is delayed and revealed that the peak of release coincides with the secondary hypoxia-induced slowing of the respiratory rhythm (Fig. 3a,b). A temporal relationship between changes in ATP concentration on the ventral surface of the medulla and hypoxia-evoked changes in the respiratory activity can be seen clearly on the expanded trace (Fig. 3b). The amount of ATP released on the ventral surface of the medulla during hypoxia in animals that were mechanically hyperventilated to induce central apnea (arterial $\mathrm{P}_{\mathrm{CO}_{2}}$ and endtidal $\mathrm{CO}_{2}$ below apneic threshold) was similar $(p>0.05)$ to that recorded during hypoxia in rats kept under normal ventilation.

An increase in ATP concentration on the ventrolateral surface of the medulla was also observed during hypoxia in sinoaortically denervated and vagotomized animals (Fig. $2 b$ ). As expected, the hypoxic respiratory response was altered in these animals, in that no increase in respiratory activity was observed (Fig. 2b). However, the secondary hypoxia-induced respiratory slowing was not affected by denervation of the peripheral chemoreceptors (Fig. $2 b$ ). Again, in these experimental conditions, an increase in ATP concentration on the ventrolateral surface of the medulla coincided with the hypoxia-evoked slowing of the respiratory rhythm (Fig. 2b). In sinoaortically denervated and vagotomized animals, the mean amplitude of the hypoxia-induced ATP release was $4.4 \pm 3.1 \mu \mathrm{M}(n=4)$, and the total amount of ATP produced was 
a

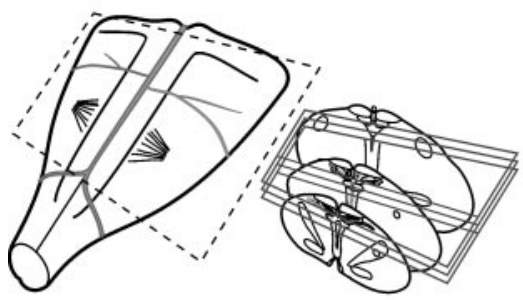

C

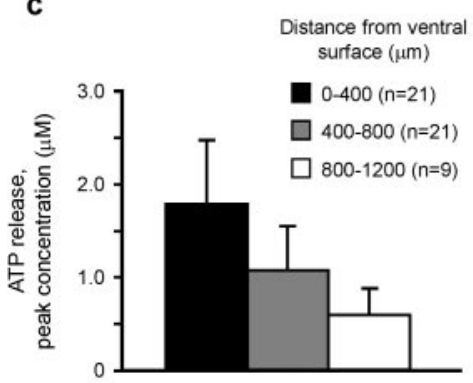

b
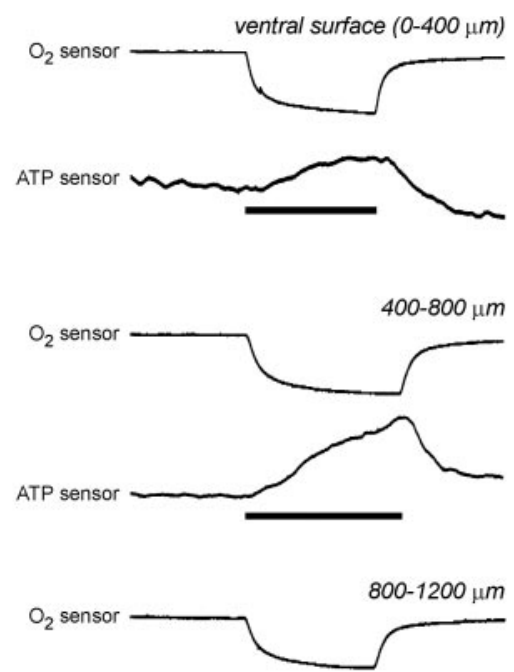

ATP sensor

Figure 4. ATP is released in the ventral medulla during hypoxia in vitro. $\boldsymbol{a}$, Schematic showing the medulla oblongata seen from the ventral surface. Three horizontal slices $(400 \mu \mathrm{m})$ were cut, from which the first one contained the ventral surface. $\boldsymbol{b}$, Raw data illustrating release of ATP from the ventral medulla during an in vitro analog of hypoxia-perfusion of the incubation chamber with aCSF in which $\mathrm{O}_{2}$ had been replaced by bubbling solution with $95 \% \mathrm{~N}_{2}$ and $5 \% \mathrm{CO}_{2}$. Note that, during hypoxia, ATP is released predominantly by the two most ventral slices of the medulla. A simultaneous null sensor exhibited no change in current during hypoxia (data not shown). c, Summary data showing the mean peak release of ATP from the horizontal slices of the medulla oblongata during hypoxia. Numbers in parentheses indicate sample sizes: in 21 experiments, we were able to examine and compare the release of ATP in the first two slices cut from the ventral surface. In nine of these experiments, we were also able to analyze the third slice from the same series.

$52.2 \pm 39.9 \mu \mathrm{M} \cdot \mathrm{s}(n=4)$. Therefore, the total amount of ATP released on the ventral surface of the medulla during hypoxia in sinoaortically denervated and vagotomized animals was significantly smaller than that recorded during hypoxia in rats with intact peripheral chemoreceptors (although the peak hypoxiainduced increase in ATP concentration was similar).

Interestingly, significant hypoxia-induced release of ATP was also recorded on the opposite surface of the medulla (Fig. 2c). An increase in ATP concentration on the dorsomedial surface of the medulla during systemic hypoxia had mean amplitude of $0.8 \pm$ $0.4 \mu \mathrm{M}(n=5)$, and the total amount of ATP produced was $315 \pm$ $182 \mu \mathrm{M} \cdot \mathrm{s}(n=5)$. The time course of ATP release on the dorsal surface of the medulla was remarkably similar to that recorded on the ventral surface (i.e., it was delayed and occurred several seconds after hypoxia-induced augmentation of the respiratory activity) (Fig. 2c).

To dissect the anatomical location of the ATP release, during hypoxia, we performed experiments in vitro on series of horizontal slices cut from the medulla oblongata (Fig. 4a). During the in vitro analog of hypoxia (saturation of aCSF with $95 \% \mathrm{~N}_{2}$ and $5 \%$ $\mathrm{CO}_{2}$ ), in 21 of 45 experiments, we recorded significant ATP release (mean amplitude, $1.7 \pm 0.7 \mu \mathrm{M} ; n=21$ ) from the most ventral slice in the series, which contained structures located $\sim 0$ $400 \mu \mathrm{m}$ from the ventral surface of the medulla oblongata (Fig. $4 b, c)$. A similar amount of ATP (mean amplitude, $1.1 \pm 0.5 \mu \mathrm{M}$; $n=21$ ) was released during hypoxia from the deeper structures of the VLM located $\sim 400-800 \mu \mathrm{m}$ from the ventral surface of the brainstem (Fig. $4 b, c$ ). Subsequent dorsal slices in the series ( $>800 \mu \mathrm{M}$ from the ventral surface) rarely exhibited a significant release of ATP (Fig. $4 b, c$ ). Thus, during hypoxia, ATP is released throughout the ventral medulla in the locations corresponding approximately to the ventral respiratory column.

\section{P2 receptor blockade in the VLM augments the hypoxia-induced secondary slowing of the respiratory rhythm}

The $\mathrm{P} 2$ receptor antagonist PPADS was microinjected unilaterally into the VLM, and the effect of this treatment on the hypoxic respiratory response was examined (Fig. 5). Microinjection of aCSF or PPADS $(100 \mu \mathrm{M})$ had no effect on baseline levels of ABP, heart rate, and phrenic nerve discharge. The baseline frequency of the phrenic nerve bursts after microinjection of aCSF or PPADS into the VLM was $39.3 \pm 3.1$ bursts/min $(n=7)$ and $36.7 \pm$ 2.5 bursts/ $\min$ ( $n=7$; not significant), respectively. The initial increase in frequency of the phrenic nerve bursts evoked by hypoxia was significantly reduced by PPADS. Thus, after microinjection of aCSF into the VLM, the hypoxia-induced peak increase in respiratory rate was $86.7 \pm 2.5$ bursts/min $(n=7)$, whereas, after microinjection of PPADS, it was $75.9 \pm 3.2$ bursts $/ \min (n=7 ; p<0.05)$. When respiratory activity was expressed as minute ventilation (respiratory rate $x$ amplitude of the phrenic nerve bursts), a slightly different picture emerged. The difference in the initial increase in minute ventilation failed to reach statistical significance $(p=0.19)$ (Fig. $5 b)$. However, the hypoxia-induced secondary slowing of the respiratory rhythm was significantly augmented by the $\mathrm{P} 2$ receptor blockade in the VLM (Fig. 5). After microinjection of PPADS, the minute ventilation was significantly lower compared with the control 2-5 min after the onset of hypoxia (Fig. $5 b$ ).

\section{Discussion}

Our use of ATP biosensors has demonstrated in vivo and in vitro that hypoxia induces a significant release of ATP at the ventral surface of the medulla and within the VLM. This ATP release is delayed; it coincides with the hypoxia-induced secondary slowing of the respiratory rhythm and partially, but not entirely, depends on inputs from peripheral chemoreceptors. Blockade of P2 receptors in the VLM potentiates the hypoxia-induced secondary slowing of the respiratory rhythm. This finding suggests that ATP, released in the medulla oblongata, does not act to initiate the hypoxic decrease in ventilation but instead helps to counteract it and maintain respiratory activity.

\section{Technical considerations}

This is one of the first in vivo studies to use an enzyme-based biosensor to monitor the release of ATP from the brain structures. In previous studies, we used biosensors to monitor adenosine release during defense reaction and systemic hypoxia in rats (Dale et al., 2002; Gourine et al., 2002). This study extends the application of the purine biosensor technology and demonstrates the feasibility of an "in vivo use" of biosensors to measure changes in ATP concentrations in the brain. 
When placed on the ventral surface of the medulla, the ATP biosensors started to respond $\sim 25$ s after hypoxia-induced augmentation of the respiratory activity. This delay could represent the time required for ATP to diffuse from the site of production and/or the response time of the ATP biosensor. The latter is unlikely, because the ATP biosensor responds immediately to changes in ATP concentration and has a $10-90 \%$ response time of $\sim 10$ s (Fig. $1 d, e)$. Thus, the sensor will quickly and accurately reflect the dynamic of changes in ATP concentration in the vicinity of the sensor. In our in vitro experiments, we observed that ATP was released during hypoxia predominantly throughout the first $\sim 800 \mu \mathrm{m}$ of the VLM as measured from the ventral surface of the brainstem. This includes significant hypoxia-induced release of ATP from the most ventral slice in the series, suggesting that the structures located in the close proximity to the ventral surface release ATP during hypoxia. If this is the case, then the ATP sensor was in direct contact with the source of ATP release in our in vivo experiments, suggesting that the latter is indeed delayed and occurs several seconds after hypoxiainduced enhancement in the respiratory activity. Diffusion of ATP from a remote site of release (from the sources located $\sim 400 \mu \mathrm{m}$ and more from the sensor) is also possible, and it would give a delayed signal at the sensor that could contribute to the overall increase in ATP concentration during hypoxia. Note that the ectonucleotidases in the medulla will cause rapid breakdown of ATP and are therefore likely to limit its diffusion to the surface. Because the sensor is insensitive to ADP or adenosine, the potential breakdown products of ATP, this would diminish the contribution of more distant dorsal sites to the sensor signal recorded at the ventral surface of the medulla.

Release of ATP in the ventral medulla during hypoxia in rats: role in hypoxic ventilatory response

The recording sites in our in vivo experiments were located $\sim 400-700 \mu \mathrm{m}$ from the ventral respiratory column, which contains a network of respiratory neurons (within the ventral respiratory groups; Bötzinger and pre-Bötzinger complexes) that are responsible for generating and shaping of the respiratory rhythm, as well as premotor neurons responsible for transmitting this rhythm to spinal motorneurons controlling the diaphragm and intercostal muscles (Duffin et al., 1995; Richter and Spyer, 2001; Feldman et al., 2003).

We and others demonstrated previously that ATP is involved in the medullary mechanisms controlling respiratory activity. $\mathrm{P}_{2} \mathrm{X}_{2}$ receptor subunits are expressed by physiologically identified respiratory neurons in the ventral respiratory column (Gourine et al., 2003). ATP increases the activity of respiratory neurons in the adult (Gourine et al., 2003) and potentiates respiratory frequency in rhythmically active in vitro preparations from neonatal rats (Lorier et al., 2004). In the latter preparation, the increase in respiratory activity evoked by $\mathrm{CO}_{2}$ does not seem to involve activation of $\mathrm{P} 2$ receptors (Lorier et al., 2004). However, the data obtained using an in vivo preparation of adult rat suggest that ATP at the level of the VLM may play an important role in central $\mathrm{CO}_{2}$ chemoreception (Spyer and Thomas, 2000; Spyer et al., 2004). Hypercapnia-induced increases in respiration are attenuated by microinjection of the $\mathrm{P} 2$ receptor antagonist suramin into the rostral VLM (Thomas et al., 1999). In addition, suramin and PPADS reduce the baseline firing and completely block hypercapnia-evoked increases in the activity of VLM neurons with inspiratory-related discharge (Thomas and Spyer, 2000). Many respiratory neurons may thus be under tonic ATP-induced excitation, which, at least in the adult, may regulate their activity during hypercapnia.

Our new results indicate that ATP plays an important role in the medullary mechanisms of the respiratory control during hypoxia as well. We suggest that, during hypoxia, ATP released in the VLM acts locally on respiratory neurons in the ventral respiratory column to maintain respiratory activity in conditions when hypoxia-induced depression of respiration occurs. During hypoxia, ATP is released at the right time, in the right place, and in sufficient quantities $(\sim 3 \mu \mathrm{M})$. Release of ATP in the VLM is delayed, and the peak of release coincides with the period of hypoxia-induced secondary slowing of the respiratory rhythm. In sinoaortically denervated and vagotomized rats, in which hypoxia induces only the depression of respiration, ATP release is still present and yet again coincides with the latter phase of respiratory depression. In vitro studies presented in Figure 4 indicate that, during hypoxia, ATP is released in the locations correspond- 
ing approximately to the ventral respiratory column. Finally, P2 receptor blockade in the VLM potentiates the hypoxia-induced secondary slowing of the respiratory rhythm.

It is important to note that $\mathrm{P} 2$ receptor antagonist PPADS was microinjected unilaterally into the rostral part of the VLM. We cannot readily estimate the rate and distance of PPADS diffusion from the injection site; however, it is very unlikely that P2 receptors in the whole ventral respiratory column have been affected. Multiple microinjections of PPADS along the rostrocaudal extent of the VLM or bilateral microinjections may have produced a greater effect on the hypoxic respiratory response; however, we were concerned that multiple injections might have compromised the neuronal function in this important area of the medulla and confounded the result of the experiment. Therefore, PPADS was injected unilaterally into the VLM. Nevertheless, this treatment still resulted in a significant augmentation of the hypoxia-induced secondary slowing of the respiratory rhythm. These results are supported by our studies on conscious $\mathrm{P}_{2} \mathrm{X}_{2}$ deficient mice using whole-body plethysmography (Rong et al., 2003). During hypoxia ( $7.5 \% \mathrm{O}_{2}$ in the breathing air), profound respiratory depression was observed in $\mathrm{P}_{2} \mathrm{X}_{2}$-deficient mice but not in their wild-type counterparts (Rong et al., 2003). These results complement the data presented here and suggest that ATP acting via $\mathrm{P} 2 \mathrm{X}_{2}$ receptors helps to counteract hypoxia-induced respiratory depression.

There is another aspect of the study conducted in our laboratory using $\mathrm{P} 2 \mathrm{X}_{2}$-deficient mice that is related to the conclusions of the present investigation. We demonstrated that, in the carotid body (primary peripheral chemosensory site), ATP is acting during hypoxia as the main transmitter to stimulate afferent terminals of the carotid sinus nerve, which convey information about arterial $\mathrm{P}_{\mathrm{O}_{2}}$ levels to the brainstem respiratory centers (Rong et al., 2003). Therefore, the actions of ATP at the two sites are synergistic: ATP in the carotid body initiates the response to hypoxia (increases ventilation), and the later release of ATP in the ventral respiratory column acts to maintain this initial response in the face of the secondary hypoxia-induced depression of respiration.

It seems that ATP release during hypoxia is not restricted to the VLM. We also observed a significant hypoxia-induced increase in ATP concentration on the dorsal surface of the medulla. Interestingly, the time course of changes in ATP concentration on the dorsal surface in relation to changes in respiration was similar to that recorded on the ventral surface. These data are in accord with our previous observation that ecto- 5 ' -nucleotidase inhibitor $\alpha, \beta$-methylene $\mathrm{ADP}$ greatly reduces the hypoxia-evoked release of adenosine at the dorsal surface, indicating the previous release of ATP (Gourine et al., 2002). Based on these data, we conclude that, at least in the medulla, the release of ATP during hypoxia could be widespread, and different medullary structures are capable of releasing ATP during hypoxic conditions.

However, the origins and mechanisms of ATP release in the medulla during hypoxia remain unknown. Both neural and nonneural sources may contribute to the increase in extracellular concentration of ATP in hypoxic conditions. It remains to be determined whether ATP is released as a cotransmitter via conventional mechanisms and/or by other routes, such as hemichannels in glia.

In any case, ATP released in the VLM during hypoxia will eventually be broken down in the extracellular space to adenosine. Several lines of evidence implicate adenosine as a mediator for hypoxia-induced depression of respiration. Hypoxic respiratory depression is reduced by the administration of adenosine receptor antagonists (Runold et al., 1989; Thomas and Marshall,
1994; Schmidt et al., 1995; Chau and Koos, 1999) or in mice lacking adenosine $A_{1}$ receptor (Johansson et al., 2001). During hypoxia, adenosine levels rise in the VLM in the cat (Richter et al., 1999). Indeed, with adenosine biosensors, we demonstrated delayed hypoxia-induced release of adenosine in the ventral and dorsal medullary regions of the rat (Gourine et al., 2002). The results of the present study show a new functional role for the ATP-mediated purinergic signaling in the medullary mechanisms controlling respiratory activity and suggest that, during sustained hypoxia, the central respiratory drive may partially depend on the balance between, respectively, the excitatory and inhibitory actions of ATP and adenosine in the ventral respiratory column.

\section{References}

Bissonnette JM (2000) Mechanisms regulating hypoxic respiratory depression during fetal and postnatal life. Am J Physiol Regul Integr Comp Physiol 278:R1391-R1400.

Chau A, Koos BJ (1999) Metabolic and cardiorespiratory responses to hypoxia in fetal sheep: adenosine receptor blockade. Am J Physiol 276:R1805-R1811.

Dale N, Pearson T, Frenguelli BG (2000) Direct measurement of adenosine release during hypoxia in the CA1 region of the rat hippocampal slice. J Physiol (Lond) 526:143-155.

Dale N, Gourine AV, Llaudet E, Bulmer D, Thomas T, Spyer KM (2002) Rapid adenosine release in the nucleus tractus solitarii during defence response in rats: real-time measurement in vivo. J Physiol (Lond) 544:149-160.

Daly M (1997) Peripheral arterial chemoreceptors and respiratorycardiovascular integration. Physiological Society Monograph Number 46. Oxford: Oxford UP.

Duffin J, Ezure K, Lipski J (1995) Breathing rhythm generation: focus on the rostral ventrolateral medulla. News Physiol Sci 10:133-140.

Feldman JL, Mitchell GS, Nattie EE (2003) Breathing: rhythmicity, plasticity, chemosensitivity. Annu Rev Neurosci 26:239-266.

Gonzalez C, Almaraz L, Obeso A, Rigual R (1994) Carotid body chemoreceptors: from natural stimuli to sensory discharges. Physiol Rev 74:829-898.

Gourine AV, Llaudet E, Thomas T, Dale N, Spyer KM (2002) Adenosine release in nucleus tractus solitarii does not appear to mediate hypoxiainduced respiratory depression in rats. J Physiol (Lond) 544:161-170.

Gourine AV, Atkinson L, Deuchars J, Spyer KM (2003) Purinergic signalling in the medullary mechanisms of respiratory control in the rat: respiratory neurones express the $\mathrm{P}_{2} \mathrm{X}_{2}$ receptor subunit. J Physiol (Lond) 552:197-211.

Johansson B, Halldner L, Dunwiddie TV, Masino SA, Poelchen W, GimenezLlort L, Escorihuela RM, Fernandez-Teruel A, Wiesenfeld-Hallin Z, Xu XJ, Hardemark A, Betsholtz C, Herlenius E, Fredholm BB (2001) Hyperalgesia, anxiety, and decreased hypoxic neuroprotection in mice lacking the adenosine $A_{1}$ receptor. Proc Natl Acad Sci USA 98:9407-9412.

Kanjhan R, Housley GD, Burton LD, Christie DL, Kippenberger A, Thorne PR, Luo L, Ryan AF (1999) Distribution of the $\mathrm{P}_{2} \mathrm{X}_{2}$ receptor subunit of the ATP-gated ion channels in the rat central nervous system. J Comp Neurol 407:11-32.

Llaudet E, Botting NP, Crayston JA, Dale N (2003) A three-enzyme microelectrode sensor for detecting purine release from central nervous system. Biosens Bioelectron 18:43-52.

Llaudet E, Droniou M, Dale N (2004) A new biosensor for the measurement of extracellular ATP in the CNS. J Physiol (Lond) 555P:C20.

Lorier AR, Peebles K, Brosenitsch T, Robinson DM, Housley GD, Funk GD (2004) P2 receptors modulate respiratory rhythm but do not contribute to central $\mathrm{CO}_{2}$ sensitivity in vitro. Respir Physiol Neurobiol 142:27-42.

North RA (2002) Molecular physiology of P2X receptors. Physiol Rev 82:1013-1067.

Paxinos G, Watson CH (1998) The rat brain in stereotaxic coordinates. London: Academic.

Prabhakar NR (2000) Oxygen sensing by the carotid body chemoreceptors. J Appl Physiol 88:2287-2295.

Richter DW, Spyer KM (2001) Studying rhythmogenesis of breathing: comparison of in vivo and in vitro models. Trends Neurosci 24:464-472.

Richter DW, Bischoff A, Anders K, Bellingham M, Windhorst U (1991) Re- 
sponse of the medullary respiratory network of the cat to hypoxia. J Physiol (Lond) 443:231-256.

Richter DW, Schmidt-Garcon P, Pierrefische O, Bischoff AM, Lalley PM (1999) Neurotransmitters and neuromodulators controlling the hypoxic respiratory response in anaesthetised cats. J Physiol (Lond) 514:567-578.

Rong W, Gourine AV, Cockayne DA, Xiang Z, Ford AP, Spyer KM, Burnstock G (2003) Pivotal role of nucleotide $\mathrm{P}_{2} \mathrm{X}_{2}$ receptor subunit of the ATPgated ion channel mediating ventilatory responses to hypoxia. J Neurosci 23:11315-11321.

Runold M, Lagercrantz H, Prabhakar NR, Fredholm BB (1989) Role of adenosine in hypoxic ventilatory depression. J Physiol (Lond) 67:541-546.

Schmidt C, Bellingham MC, Richter DW (1995) Adenosinergic modulation of respiratory neurones and hypoxic responses in the anaesthetized cat. J Physiol (Lond) 483:769-781.

Spyer KM, Thomas T (2000) Sensing arterial $\mathrm{CO}_{2}$ levels: a role for medullary P2X receptors. J Auton Nerv Syst 81:228-235.

Spyer KM, Dale N, Gourine AV (2004) ATP is a key mediator of central and peripheral chemosensory transduction. Exp Physiol 89:53-59.

Thomas T, Marshall JM (1994) Interdependence of respiratory and cardio- vascular changes induced by systemic hypoxia in the rat: the roles of adenosine. J Physiol (Lond) 480:627-636.

Thomas T, Spyer KM (2000) ATP as a mediator of mammalian central $\mathrm{CO}_{2}$ chemoreception. J Physiol (Lond) 523:441-447.

Thomas T, Ralevic V, Gadd CA, Spyer KM (1999) Central $\mathrm{CO}_{2}$ chemoreception: a mechanism involving $\mathrm{P} 2$ purinoceptors localized in the ventrolateral medulla of the anaesthetized rat. J Physiol (Lond) 517:899-905.

Thomas T, Ralevic V, Bardini M, Burnstock G, Spyer KM (2001) Evidence for the involvement of purinergic signalling in the control of respiration. Neuroscience 107:481-490.

Yao ST, Barden JA, Finkelstein DI, Bennett MR, Lawrence AJ (2000) Comparative study on the distribution patterns of $\mathrm{P} 2 \mathrm{X}_{1}-\mathrm{P} 2 \mathrm{X}_{6}$ receptor immunoreactivity in the brainstem of the rat and the common marmoset (Callithrix jacchus): association with catecholamine cell groups. J Comp Neurol 427:485-507.

Yao ST, Gourine AV, Spyer KM, Barden JA, Lawrence AJ (2003) Localisation of $\mathrm{P} 2 \mathrm{X}_{2}$ receptor subunit immunoreactivity on nitric oxide synthase expressing neurones in the brain stem and hypothalamus of the rat: a fluorescence immunohistochemical study. Neuroscience 121:411-419. 